\title{
Anomalous Fluid Transport in Porous Media Induced by Biofilm Growth
}

\author{
Joseph D. Seymour, ${ }^{1,2}$ Justin P. Gage, ${ }^{1,2}$ Sarah L. Codd, ${ }^{1,2}$ and Robin Gerlach ${ }^{2}$ \\ ${ }^{1}$ Department of Chemical and Biological Engineering, Montana State University, Bozeman, Montana 59717-3920, USA \\ ${ }^{2}$ Center for Biofilm Engineering, Montana State University, Bozeman, Montana 59717-3980, USA \\ (Received 27 August 2004; published 3 November 2004)
}

\begin{abstract}
Magnetic resonance measurements of the transition from normal to anomalous hydrodynamic dispersion in porous media due to biological activity are presented. Fractional advection-diffusion equations are shown to provide models for the measured impact of biofilm growth on porous media transport dynamics.
\end{abstract}

DOI: 10.1103/PhysRevLett.93.198103

The impact of microbial activity, particularly surface attached biofilms, on the transport of fluids in porous systems is relevant to fields as seemingly diverse as geophysics and medicine [1]. Few direct experimental data on the impact of bioactivity on transport dynamics in three-dimensional media are available due to sample opacity. Noninvasive magnetic resonance microscopy (MRM) [2] directly measures length and time scale dependent dynamics in porous media [3,4]. Here we show by direct measurement of the propagator, i.e., the displacement conditional probability [5] or van Hove scattering function [2], the transition from normal to anomalous hydrodynamic dispersion as a function of bioactivity. Microbial activity transforms the porous media from a homogeneous to a heterogeneous structure, increasing system complexity as defined in terms of dynamics [6]. Continuous time random walk (CTRW) based fractional advection-diffusion equation (ADE) models which generate anomalous or fractional dynamics [7-9] are compared to the measured dynamics in both the propagator displacement space and the Fourier reciprocal displacement wavelength space. The data provide insight into the application and development of fractional calculus based models and indicate their direct applicability to biofilm impacted porous media transport.

In porous media such as Earth's subsurface microbial biofilms modify permeability and can serve to enhance oil recovery or alter contaminant transport in bioremediation. In medical applications microbial fouling of porous and gel based biomedical devices, such as filtration systems, is pertinent to infection and system function and design. Pulsed gradient spin echo (PGSE) magnetic resonance (MR) methods have found significant application in the study of transport in model and natural porous media due to the ability to measure molecular dynamics in opaque systems $[10,11]$. The characterization of porous media structure by molecular diffusion [5] and bulk flow $[12,13]$ is well established as is the ability to study displacement time and wavelength dependence of hydrodynamic dispersion $[4,12,14]$. The study of biofilm impact on three-dimensional porous media transport dynamics has been limited to bulk measurements of pressure drop
PACS numbers: 87.10.+e, 05.40.Fb, 05.60.-k, 92.40.Cy

correlated to permeability [15]. MR methods have been applied to study the spatial distribution of biofilms in porous media by diffusion [16] and magnetic relaxation time weighting [17] methods; however, to our knowledge, no detailed study of the impact on scale dependent dispersion dynamics has been undertaken.

Anomalous transport processes, those in which the mean squared displacement varies nonlinearly in time and which do not obey Gaussian statistics, are found in many physical, chemical, and biological systems due to the presence of correlated dynamics $[7,8,18]$. CTRW theory provides a basis for modeling anomalous transport $[7,8,18]$ and is equivalent to a mass or probability conservation approach based on generalized master equations [19] from which fractional ADE's can be derived [20]. The fractional ADE is consistent with nonlocal continuum mechanics [21] and nonequilibrium statistical mechanics [22] models for transport in heterogeneous porous media, i.e., those with property variations over a hierarchy of scales. Fractional dynamics based on CTRW's represents a powerful extension of macroscale transport [23] modeling in complex systems [6], by inclusion of memory effects [24]. CTRW's characterize transport in terms of a jump probability distribution $\psi(Z, t)$ for a tracer particle to undergo a jump $Z$ after waiting time $t$. Transport equations which are fractional in either space or time are derived by assuming independence of the jump length, $\lambda(Z)$, and waiting time, $w(t)$, distributions so that $\psi(Z, t)=\lambda(Z) w(t)$. Normal diffusion, or Brownian motion, is recovered for finite mean waiting times and jump length variances. Anomalous diffusion is obtained in the form of a time fractional ADE for divergent mean waiting time and a space fractional ADE for divergent jump length variances, corresponding to power law wait time and jump length distributions $[8,20]$.

MRM images of the impact of biofilm growth on the pore scale velocity and magnetic relaxation time in a model porous media constructed of $241 \mu \mathrm{m}$ monodisperse (Duke Scientific 4324A) beads for fixed volumetric flow rate are shown in Fig. 1. The pore scale velocity images indicate greater spatial variability of the velocity 


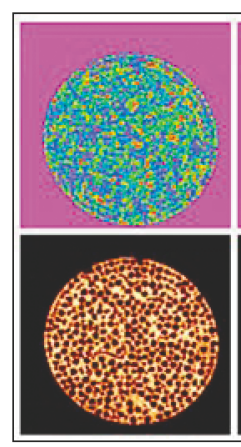

Day 1

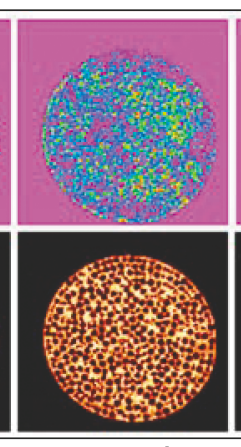

Day 2

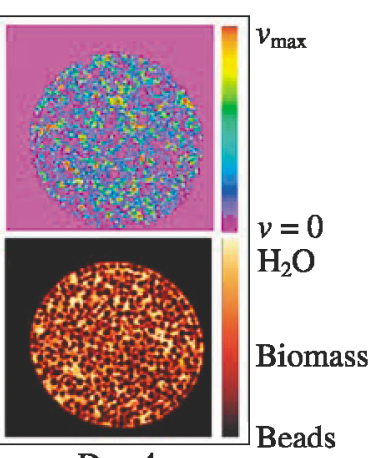

Day 4
FIG. 1 (color). MRM maps of velocity (top row) and $T_{2}$ magnetic relaxation (bottom row) as a function of biofilm growth time (left to right). Day 1 shows the clean porous media composed of $241 \mu \mathrm{m}$ diameter monodisperse polystyrene beads packed in a $5 \mathrm{~mm}$ i.d. cylinder. Spatial resolution is $54.7 \mu \mathrm{m} /$ pixel $(128 \times 128$ pixels $)$ in plane, so the data reflect pore scale spatial distributions of velocity over a $1000 \mu \mathrm{m}$ slice and biomass over a $200 \mu \mathrm{m}$ slice. The biomass is just discernible by day 2 in the $T_{2}$ map, while the velocity distribution shows a flow composed of regions of no flow and high velocity. By day 4 biomass is clearly evident in the $T_{2}$ map and the velocity distribution shows more regions of no flow and even higher velocities.

due to biofilm growth 2 and $4 \mathrm{~d}$ after inoculation with Pseudomonas aeruginosa (FRD1 containing the plasmid pAB1). Increased regions of no flow due to biomass blocked pores generate high velocity in unclogged pores to conserve mass for the fixed flow rate. The transverse magnetic relaxation time images indicate the spatial distribution of the biomass growth by measuring enhanced relaxation due to the restricted motion of water molecules within the biomass [17]. On day 2 the impact on pore scale velocity is more significant than on the magnetic relaxation, demonstrating the sensitivity of transport processes to small amounts of biofilm growth.

To quantify the impact of the biofilm growth, PGSE MR is used to measure the propagator, or conditional probability, $P_{s}\left(z \mid z^{\prime}, t\right)$ of a displacement from $z$ to $z^{\prime}$ over time $t$. In PGSE MR a pair of pulsed magnetic field gradients is applied so as to encode in the phase of the magnetization the location of all MR active spins at an initial time and then unwind that phase at a set time $\Delta$ later, generating phase shifts dependent on molecular dynamics over time $\Delta[2,5]$. The measured echo signal $E(q, \Delta)$ is the Fourier inversion of the propagator averaged over the initial spin density $\rho(z)$,

$$
\begin{aligned}
E(q, \Delta) & =\iint \rho(z) P_{s}\left(z \mid z^{\prime}, \Delta\right) \exp \left[i 2 \pi q\left(z^{\prime}-z\right)\right] d z d z^{\prime} \\
& =\int P(Z, \Delta) \exp (i 2 \pi q Z) d Z
\end{aligned}
$$

where $q=(2 \pi)^{-1} \gamma g \delta$ is the Fourier reciprocal wavelength to displacement $Z=z^{\prime}-z$ and $P(Z, \Delta)$ the aver- aged propagator [2]. Figure 2(a) shows the measured propagator transition for flow at $1.67 \mathrm{ml} / \mathrm{min}$, Peclet number of 270, through the clean porous media from preasymptotic at $\Delta=20 \mathrm{~ms}$ to Gaussian dynamics at $\Delta=300 \mathrm{~ms}$. Figure 2(b) displays the measured propagators for an observation time $\Delta=300 \mathrm{~ms}$ as a function of biofilm growth for the same fixed volumetric flow rate. The day 1 clean porous media propagator is Gaussian, indicating normal transport. The growth of the biofilm, day 3 and day 7, alters the dynamics with increased probability of small displacements due to fluid which is entrained in the biomass and trapped in clogged dead-end pores and persistent tails in the distribution due to higher probability of large displacements in high permeability unclogged channels. The data visualize an increase in the complexity of the porous media due to bioactivity in the sense defined by Goldenfeld and Kadanoff [6], a divergence from normal transport statistics. The homogeneous clean porous media have uniform pore size and permeability which generate normal Gaussian dynamics. The biofilm growth induces a heterogeneous structural tran-
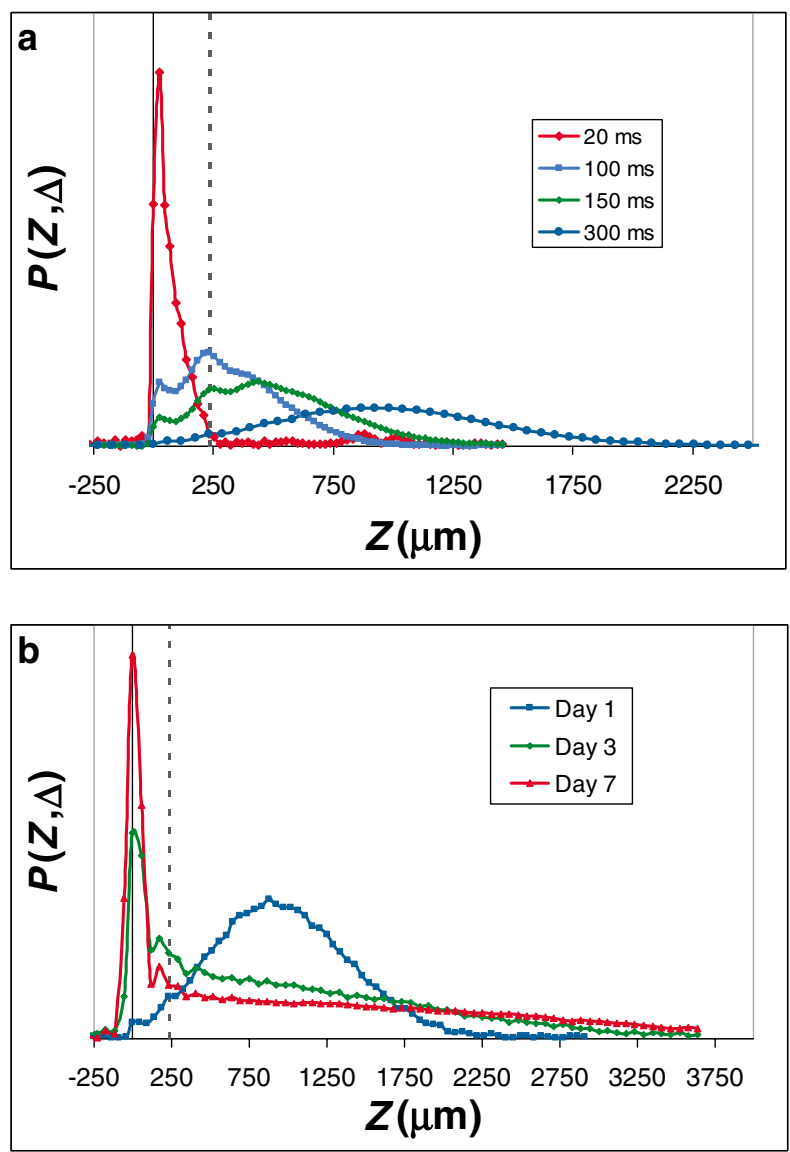

FIG. 2 (color). Measured propagators for (a) the transition from preasymptotic to asymptotic Gaussian dynamics in the clean bead pack as a function of observation time $\Delta$ and (b) the transition from Gaussian to anomalous dynamics induced by biofilm growth for an observation time $\Delta=300 \mathrm{~ms}$. The vertical dashed lines are the bead diameter. 
sition with variable pore sizes and permeability variations over a range of length scales that generate a nonGaussian displacement distribution.

The CTRW formalism provides a model for the transition shown in Fig. 2(b) based on the strongly varying spatial distribution of velocity shown in Fig. 1. Molecules trapped in the biomass or fluid in blocked and partially blocked pores must diffuse or slowly flow out to join the faster moving fluid and have long wait times, while molecules in minimally blocked or clean pores have shorter or nonexistent wait times. The conceptual model is that a molecular jump is independent of the preceding wait time but depends on the velocity at the starting point $[20,24]$, in our case the position at the time of the first gradient pulse of the PGSE pair.

A power law Lévy stable wait time distribution, $w(t) \sim$ $t^{-1-\alpha}$, for $0<\alpha<1$ leading to divergent mean wait time and a Gaussian jump length distribution with finite variance and starting velocity dependent shift are applied $[8,20,24]$. The result is the time fractional ADE, or fractional Fokker-Planck equation,

$$
\begin{aligned}
\frac{\partial P\left(Z^{*}, t^{*}\right)}{\partial t^{*}}= & { }_{0} \mathbf{D}_{t}^{1-\alpha}\left[-A_{\alpha} \frac{\partial}{\partial Z^{*}} v\left(Z^{*}\right)\right. \\
& \left.+D_{\alpha} \frac{\partial^{2}}{\partial Z^{* 2}}\right] P\left(Z^{*}, t^{*}\right),
\end{aligned}
$$

where $A_{\alpha}$ is a derivation derived advection parameter and $D_{\alpha}$ is the effective diffusion, or dispersion coefficient $[8,20,24]$. The fractional Riemann-Liouville operator is defined as

$$
{ }_{0} \mathbf{D}_{t}^{1-\alpha} P\left(Z^{*}, t^{*}\right)=\frac{1}{\Gamma(\alpha)} \frac{\partial}{\partial t^{*}} \int_{0}^{t^{*}} \frac{P\left(Z^{*}, t^{*}\right)}{\left(t^{*}-t^{\prime}\right)^{1-\alpha}} d t^{\prime} .
$$

Note that for $\alpha=1$ the Riemann-Liouville operator is unity and Eq. (2) becomes the normal ADE and Gaussian statistics are obtained. Plots of $P\left(Z^{*}, t^{*}=3\right)$ for a constant velocity, generated using the scaling relation between the fractional and Brownian solutions [8,24], are shown in Fig. 3. Note the transition from the Galilei invariant Gaussian for $\alpha=1$ to the Galilei variant forms for decreasing $\alpha$ and the increase in the long displacement tail in qualitative agreement with the biofilm induced transition. The biofilm growth induces a transition to fractional dynamics.

The measurement of anomalous transport by PGSE MR methods [25] has been limited primarily to the fractional time dependence of the variance, $\left\langle Z(t)^{2}\right\rangle \sim D_{\kappa} t^{\kappa}$, using the well known scaling [26] $E(q, \Delta) \sim$ $\exp \left(-4 \pi^{2} q^{2} D_{\kappa} \Delta^{\kappa}\right)$. Recent theoretical predictions indicate diffusion in fractal dimension, $d_{f}$, systems should generate a power law scaling, $E(q, \Delta) \sim q^{2-d_{f}}$ in the long wavelength limit [27]. Stretched exponential wavelength scaling, $E(q, \Delta) \sim \exp \left(-c q^{\beta}\right)$, has been applied in a phenomenological fashion to PGSE MR data for biological tissues [28] and corresponds to a space fractional model

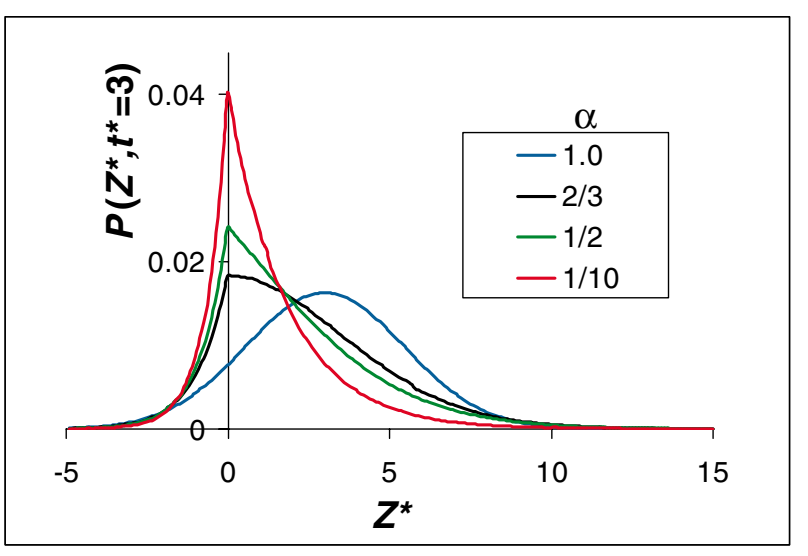

FIG. 3 (color). Theoretical propagators generated for solution of Eq. (3), the time fractional ADE. The normal ADE is recovered for $\alpha=1$ and transitions to stronger subdiffusive behavior for decreasing $\alpha$, with the peak increasing around $Z^{*}=$ 0 . Note the Galilei invariance of the normal ADE; i.e., distribution is translated by the mean velocity. In contrast, the fractional model is Galilei variant and the distribution is skewed. The graph is generated numerically using the scaling relationship between the fractional and Brownian solutions [8,24].

[8]. Analysis of the data in $q$ space [5], Fig. 4(a), provides additional interpretive detail of the biofilm impact on the hydrodynamic dispersion dynamics [12]. The day 1 data for the clean bead pack show rapid Gaussian decay followed by a flow induced diffractionlike effect demonstrating the regular pore size $[3,12,13]$. In contrast, the biofilm data, days $2,3,5$, and 7 , no longer exhibit the rapid Gaussian decay and show the transition from a structurally ordered homogeneous porous media which induce a diffractionlike signal modulation to a more complex [6] heterogeneous structure.

The power law nature of the tails of the propagators in Fig. 2 is quantified by the parameter $\beta$ in stretched exponential, $E(q, \Delta) \sim \exp \left(-c q^{\beta}\right)$, fits of the low- $q$ data [7], $q<\left(d_{\text {bead }}\right)^{-1}$, as a function of observation time, Fig. 4(b). This corresponds to a fractional ADE model with a Poisson wait time distribution and a Lévy distribution for the jump length [8]. The preasymptotic transition in Fig. 2(a) from a Cauchy-like to a Gaussian distribution for the clean porous media involves a transition in $q$-space scaling, Fig. 4(b), from exponential at short times, $\beta \sim 1$, to Gaussian, $\beta \sim 2$, in the asymptotic limit. Note the difference in the asymptotic $\beta$ for the two runs due to packing differences. The biofilm impacted dispersion data have decreasing $\beta$ with increasing observation time. The stretched exponential in low $q$ space corresponds to the power law behavior of the propagator tails since the Fourier relationship provides the scaling $P(Z, \Delta) \sim Z^{(1+\beta)}$, and the transition in $\beta$ at $\Delta=300 \mathrm{~ms}$ from $\sim 2$ to $\sim 0.4$ due to biofilm growth quantifies the transition to a scale invariant distribution [7].

We find that biofilm growth in porous media generates a transition in the hydrodynamic dispersion dynamics 

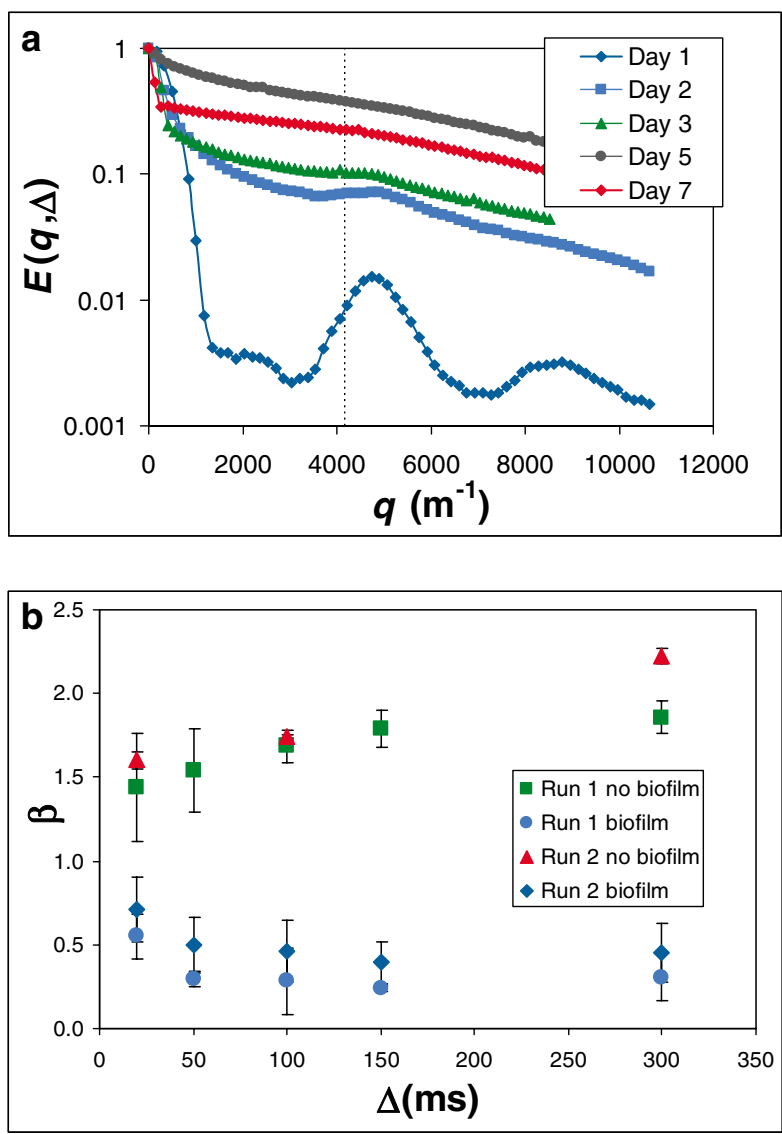

FIG. 4 (color). Biofilm induced transition of porous media dynamics analyzed in the Fourier reciprocal $q$ space [5]. (a) The echo signal shows the transition due to biofilm growth from day 1 clean porous media, a rapid Gaussian decay with diffractionlike coherence feature minima at the reciprocal bead diameter, to a stretched exponential behavior at later days. Days 1, 3, and 7 data correspond to Fig. 2(b). Note day 5 has the most biomass present and a sloughing event occurred between day 5 and day 7. (b) Stretched exponential, $E(q, \Delta) \sim$ $\exp \left(-c q^{\beta}\right), q$ scaling parameter $\beta$ as a function of displacement time $\Delta$ in the clean and biofilm impacted porous media. Scaling in the clean bead pack increases toward a Gaussian, $\beta \sim 2$, with increasing time, while the biofilm generates dynamics with decreasing $\beta$. Values of $\beta$ at each $\Delta$ are averages of multiple PGSE NMR experiments over $7 \mathrm{~d}$ for two separate biofilm runs and the error bars are standard deviation.

which can be modeled as a transition from normal to anomalous transport using a fractional ADE. The strong impact of the biofilm on flow dynamics coupled with recent innovations in MR technology for inexpensive low magnetic field applications provides for the concept of online biofilm assays [16] for bioreactor and filtration technology. The data analysis shows strong coupling between the bioactivity induced evolution of the propagator and stretched exponential scaling in $q$ space providing a basis for further exploration of the coupling of space and time [19] in CTRW based fractional models. Fractional ADE models represent a powerful new tool for predictive modeling of complex biophysical systems and continued theoretical development, and comparison to measurements will provide the refinement necessary for broad application.

This research was supported by Office of Science (BER), U.S. Department of Energy DE-FG0203ER63576, National Science Foundation CTS-0348076 (J. D. S.), DMI-0340709 (S. L. C.), Inland Northwest Research Alliance (J.P.G.), and ACS Petroleum Research Fund. The authors thank R. Metzler for a comment on the generation of Fig. 3.

[1] J.W. Costerton and P. S. Stewart, Sci. Am. 285, 1 (2001).

[2] P.T. Callaghan, Principles of Nuclear Magnetic Resonance Microscopy (Oxford University Press, New York, 1991).

[3] J. D. Seymour and P. T. Callaghan, J. Magn. Reson., Ser. A 122, 90 (1996).

[4] A. Ding and D. Candela, Phys. Rev. E 54, 656 (1996).

[5] P. T. Callaghan et al., Nature (London) 351, 467 (1991).

[6] N. Goldenfeld and L.P. Kadanoff, Science 284, 87 (1999).

[7] M. F. Shlesinger, G. M. Zaslavsky, and J. Klafter, Nature (London) 363, 31 (1993).

[8] R. Metzler and J. Klafter, Phys. Rep. 339, 1 (2000).

[9] B. Berkowitz et al., Water Resour. Res. 38, 1191 (2002).

[10] H. van As and P. Lens, J. Ind. Microbiol. Biotechnol. 26, 43 (2001).

[11] L. F. Gladden, AIChE J. 49, 2 (2003).

[12] J. D. Seymour and P.T. Callaghan, AIChE J. 43, 2096 (1997).

[13] B. Manz, P. Alexander, and L. F. Gladden, Phys. Fluids 11, 259 (1999).

[14] S. Stapf et al., Phys. Rev. E 58, 6206 (1998).

[15] A. B. Cunningham et al., Environ. Sci. Technol. 25, 1305 (1991).

[16] K. Potter et al., J. Magn. Reson., Ser. B 113, 9 (1996).

[17] B. C. Hoskins et al., J. Magn. Reson., Ser. B 139, 67 (1999).

[18] J.-P. Bouchaud and A. Georges, Phys. Rep. 195, 127 (1990).

[19] V. M. Kenkre, E.W. Montroll, and M. F. Shlesinger, J. Stat. Phys. 9, 45 (1973).

[20] A. Compte, Phys. Rev. E 55, 6821 (1997).

[21] D. L. Koch and J. F. Brady, J. Fluid Mech. 180, 387 (1987).

[22] J. H. Cushman and T. R. Ginn, Water Resour. Res. 36, 3763 (2000).

[23] H. Brenner, J. Stat. Phys. 62, 1095 (1991).

[24] R. Metzler and A. Compte, J. Phys. Chem. B 104, 3858 (2000).

[25] R. Kimmich, Chem. Phys. 284, 253 (2002).

[26] J. Karger, H. Pfeifer, and G. Vojta, Phys. Rev. A 37, 4514 (1988).

[27] R. A. Damion and K. J. Packer, Proc. R. Soc. London A 453, 205 (1997).

[28] M. Kopf et al., Biophys. J. 70, 2950 (1996). 